\title{
Application Value of Combined Diagnosis of Ultrasound, MRI, and $X$-Ray in Developmental Dysplasia of the Hip in Children
}

\author{
Jian Li, ${ }^{1}$ Bo Zhao, ${ }^{1}$ Honghua Ji, ${ }^{1}$ and Wei Ding $\mathbb{D D}^{2}$ \\ ${ }^{1}$ Department of Ultrasonography, Binzhou Hospital of Traditional Chinese Medicine, Binzhou 256601, Shandong, China \\ ${ }^{2}$ Department of Ultrasonography, People's Hospital of Rizhao, Rizhao 276826, Shandong, China \\ Correspondence should be addressed to Wei Ding; dingwei@sdrzph.cn
}

Received 2 December 2021; Revised 16 December 2021; Accepted 4 January 2022; Published 19 January 2022

Academic Editor: Yuvaraja Teekaraman

Copyright (C) 2022 Jian Li et al. This is an open access article distributed under the Creative Commons Attribution License, which permits unrestricted use, distribution, and reproduction in any medium, provided the original work is properly cited.

Objective. To explore the application value of the combined diagnosis of ultrasound, MRI, and X-ray in developmental dysplasia of the hip (DDH) in children. Methods. Ninety children with suspected DDH admitted to our hospital from June 2017 to June 2020 were selected as the research objects to conduct a retrospective study. According to the age of the children, they were divided into a group with 0-6 months (group X), a group with 7-12 months (group Y), and a group older than 12 months (group Z), with 30 cases in each group. X-ray and high-frequency ultrasound were performed in all groups, and MRI examination was added to the children in groups $\mathrm{Y}$ and $Z$ to compare the diagnostic value of the three imaging examinations in DDH children. Results. No obvious differences in the general data and maternal risk factors were observed among the three groups $(P<0.05)$. The final comprehensive diagnostic results were taken as the gold standard, including 23 cases with acetabular dysplasia, 28 cases with subluxation of the femoral head, 31 cases with complete dislocation of the femoral head, and 8 non-DDH cases. The diagnostic accuracy of the three methods from high to low was MRI, high-frequency ultrasound, and X-ray, with obviously higher diagnostic accuracy of MRI than that of X-ray $(P<0.05)$. The ROC curves showed that the diagnostic efficacy from high to low was $\mathrm{MRI}+$ high-frequency ultrasound $+\mathrm{X}$-ray, high-frequency ultrasound $+\mathrm{X}$-ray, MRI, high-frequency ultrasound, and X-ray. Conclusion. Ultrasound combined with X-ray has obvious advantages in the diagnosis of children at low months of age, while MRI has outstanding advantages in the diagnosis of children at high months of age. MRI combined with ultrasound and X-ray can significantly improve the diagnostic accuracy of DDH and provide objective data support for the clinical treatment of children.

\section{Introduction}

Developmental dysplasia of the hip (DDH) in children is the most common hip disease in pediatric orthopedics. With complex pathological structure and dynamic development, DDH mainly includes acetabular dysplasia, subluxation of the femoral head, and complete dislocation of the femoral head [1-4]. According to clinical observation, early diagnosis and treatment are crucial for DDH children, and early diagnosed children can achieve satisfactory results under timely conservative treatment. Otherwise, the growth of children will not only increase the difficulty of treatment but also reduce the efficacy. Nowadays, still occupying a large proportion, many DDH children do not receive timely treatment. These children may have diseases such as gait abnormalities and spinal abnormalities and may be prone to long-term and chronic degenerative hip diseases in adulthood, accompanied by long-term pain, or even disability in severe cases [5-8]. It can be seen that early diagnosis is the key to guiding clinical decision-making and obtaining good prognosis, and imaging examination is the main method to determine DDH at present. Among them, ultrasound, X-ray, and MRI are the main methods for clinical diagnosis of DDH in children. There are many studies on the three examination methods applied in the diagnosis of $\mathrm{DDH}$, but no unified and effective imaging diagnosis scheme has been formed. Based on this, this study explored the application value of MRI combined with X-ray and ultrasound in the diagnosis of DDH in children. 


\section{Study Protocol}

2.1. Case Screening. The inclusion criteria for children were formed as follows according to research objectives and procedures. (1) Children were initially diagnosed as suspected DDH cases according to routine physical examination; (2) children had one or more of the following signs and symptoms: (a) asymmetric hip or thigh patterns, (b) unilateral hip joint retraction or bilateral dislocation and widened perineum, (c) positive Allis sign, (d) positive result in left abduction test, (e) positive Ortolani sign, (f) positive Barlow sign, (g) less limb movement and weak pedaling in children, and (h) the hip joint had clicking; (3) children were no more than 12 years old; (4) children had complete medical records; (5) the family members of the children were informed of and agreed to this study; and (6) children received the imaging examinations for the first time.

2.1.1. Exclusion Criteria. (1) Children had malformed and developmental dislocation of the hip; (2) before enrollment, children did not receive the imaging examinations required for this study; (3) children had secondary joint dislocation; and (4) children had multiple joint contracture or cerebral palsy.

According to the above criteria, 90 children with suspected DDH admitted to our hospital from June 2017 to June 2020 were selected for a retrospective study.

2.2. Grouping. According to their age, the 90 enrolled children were divided into a group with 0-6 months (group $\mathrm{X}$ ), a group with 7-12 months (group Y), and a group older than 12 months (group Z), with 30 cases in each group. The hospital ethics committee approved the study and supervised the implementation of the study protocol.

2.3. Methods. X-ray and high-frequency ultrasound were performed in all groups, and MRI examination was added to the children in groups $\mathrm{Y}$ and $\mathrm{Z}$. Sedation was needed during MRI examination, so MRI was not suitable for low-month children.

2.3.1. X-Ray Examination. The examiner helped the child lie quietly on his back, with the distance between his lower limbs as wide as his shoulder breadth, and the tiptoes rotated about $20^{\circ}$ inward. The Primary Diagnost DR machine (manufacturer: Philips) was used for scanning, and the anteroposterior radiographs of the hip were taken to obtain clear X-ray films. According to the parameters such as acetabular angle, central-edge angle, Perkin square, Calve line, and Shonton line, the acetabular development of the children was determined [9-12].

2.3.2. High-Frequency Ultrasound Examination. The child stayed lateral, with the hip joints buckled to $30^{\circ}$. The examiner used a color Doppler ultrasound diagnosis apparatus (model: DC-N2S) with a linear array probe of $5-7.5 \mathrm{MHz}$ to scan the hip joints of the child and to obtain the coronal plane of femoral greater trochanter. Then, the probe was perpendicular to the scanning plane, and continuous scanning was performed. The coronal plane images were obtained by parallel scanning from the back to the ventral part. The first line (baseline) was drawn from the top of the acetabular cartilage to the lateral line that was tangential to the iliac bone plate. The lower edge of the iliac bone in the acetabular fossa was connected with the external and lower angle of the acetabular bone, as the second line. The midpoint of the labrum articularis was connected with the external and lower angle of the acetabular bone, as the third line. The angle between the first and second lines was $\alpha$, and the angle between the second and third lines was $\beta$ [12-15].

2.3.3. MRI Examination. The selected instrument was $3.0 \mathrm{~T}$ superconducting Avanto magnetic resonance instrument (manufacturer: Siemens). Appropriate sedation could be given to the child who could not cooperate with the examiner, and MRI examination was performed after the child was asleep. The child was supine, with both lower limbs straight and the tiptoes buckled. Small soft pads were placed under both ankle joints, and the ankle and knee joints were tied with fixing straps. Abdominal coil scanning was performed to fully cover both bilateral hip joints and the distal femur. Phase scanning was performed according to the order of axial, coronal, and sagittal positions, and then the scanning line was scanned in parallel with the femoral neck axis [16-18]. Scanning with the $\mathrm{T}_{2}$ TSE sequence was performed to obtain the oblique sagittal image of the femoral neck. The center of the femoral head was connected with the midpoint of the narrowest part of the femoral neck to obtain the femoral neck axis, which formed a $Q$ angle with the horizontal line. The distal femur of the affected side was scanned with the $\mathrm{T}_{1} \mathrm{TSE}$ sequence to obtain the axial image. The condyle line was formed by connecting the posterior margin of the epiphyseal cartilage of the internal and external condyles, forming a $B$ angle with the horizontal line. The anteversion of femoral neck was calculated according to angles $Q$ and $B$.

\subsection{DDH Diagnostic Criteria}

2.4.1. X-Ray Examination. Acetabular dysplasia could be determined with acetabulum angle $>30^{\circ}$, center-edge angle $<20^{\circ}$, discontinuous Shonton line, discontinuous Calve line, and Perkin square in the outer lower or outer upper quadrant.

2.4.2. High-Frequency Ultrasound. (1) Complete hip dislocation: $\alpha<45^{\circ}$ and $\beta$ not available. (2) Hip subluxation: $45^{\circ}<\alpha \leq 50^{\circ}$ and $\beta>77^{\circ}$. (3) DDH: $50^{\circ}<\alpha \leq 55^{\circ}$ and $55^{\circ} \leq \beta \leq 77^{\circ}$. (4) Hip instability: $55^{\circ}<\alpha \leq 60^{\circ}$ and $55 \leq \beta \leq 77^{\circ}$. (5) Normal hip: $\alpha>60^{\circ}$ and $\beta<55^{\circ}$.

2.4.3. MRI Examination. DDH was judged according to the age of the children and the anteversion of femoral neck. The normal anteversion of femoral neck was $25^{\circ}-35^{\circ}$, which 
decreased by $1^{\circ}$ per one-year increase of age, and children beyond this range could be diagnosed with DDH.

2.5. Statistical Treatment. The data obtained in this study were processed by software SPSS22.0 and graphed by software GraphPad Prism 7 (GraphPad Software, San Diego, USA). The study data included enumeration data and measurement data, expressed as $n(\%)$ and $(\bar{x}+s)$ and tested by $X^{2}$ and $t$-test. The differences were statistically significant at $P<0.05$.

\section{Results}

3.1. General Data. No obvious differences in the general data and maternal risk factors were observed among the three groups $(P<0.05)$ (see Table 1$)$.

3.2. Clinical Diagnostic Results. The final comprehensive diagnostic results were taken as the gold standard, including 23 cases with acetabular dysplasia, 28 cases with subluxation of the femoral head, 31 cases with complete dislocation of the femoral head, and 8 non-DDH cases, as presented in Table 2.

3.3. Diagnostic Results of X-Ray, Ultrasound, and MRI. The diagnostic accuracy of the three methods from high to low was MRI, high-frequency ultrasound, and X-ray, with obviously higher diagnostic accuracy of MRI than that of $\mathrm{X}$-ray $(P<0.05)$ (see Figure 1$)$.

3.4. ROC Curves. The ROC curves showed that the diagnostic efficacy from high to low was MRI + high-frequency ultrasound + X-ray, high-frequency ultrasound + X-ray, MRI, high-frequency ultrasound, and X-ray, as shown in Figure 2. The area under the curves is shown in Table 3.

\section{Discussion}

Imaging examination, an important basis for early diagnosis, treatment, and follow-up after intervention of DDH, can directly reflect the hip structure and development of children [19-22]. Since DDH mostly occurs in infants and young children, the subjects for examination are special. In addition, early diagnosis, early treatment, and timely adjustment of the treatment plans play a vital role in the later growth of the children. Therefore, the clinical diagnosis of $\mathrm{DDH}$ is inseparable from the support of imaging data from high-frequency ultrasound, X-ray, and MRI. Though there are many related comparative studies at present, these three methods are highly flexible when applied in the diagnosis of infants and young children with DDH, and no complete and effective application plan has been formed. Based on this, this paper explored the application value of the combined diagnosis of ultrasound, MRI, and X-ray in DDH children and tried to explore a feasible scheme for clinical diagnosis and treatment of DDH. In this study, no obvious differences in the general data and maternal risk factors were observed among the three groups $(P<0.05)$. The general data of children were recorded, and the influencing factors that may lead to DDH in children were analyzed. It was found that children in each group were accompanied by different levels of risk factors, such as high birth weight, multiple pregnancy, and oligohydramnios, which were consistent with the clinical status. Therefore, in early screening of DDH newborns, hospitals should strengthen the awareness of the above risk factors to improve the clinical screening rate of neonatal DDH. The final comprehensive diagnostic results were taken as the gold standard, including 23 cases with acetabular dysplasia, 28 cases with subluxation of the femoral head, 31 cases with complete dislocation of the femoral head, and 8 non-DDH cases. The diagnostic accuracy of the three methods from high to low was MRI, high-frequency ultrasound, and X-ray, with obviously higher diagnostic accuracy of MRI than that of X-ray $(P<0.05)$. These results were in line with the study of Walbron [23], suggesting that MRI, ultrasound, and X-ray had certain advantages in the diagnosis of DDH in children. Firstly, DDH usually occurs in newborns or infants, and children with low months of age have not yet shown ossific hip joints. Therefore, ultrasound shows high superiority in the diagnosis of DDH children with low months, and it can be used as the preferred standard for DDH screening in these children, especially for high-risk children. Alassaf et al. [24] have reported that, for newborns with mild dysplasia but stable hips shortly after birth, treatment is not required, but active ultrasonic monitoring is necessary. Secondly, X-ray examination is fast and economic. However, the ossification center of femoral head has not yet formed in infants within 3 months, and indicators such as Perkin quadrant, center-edge angle, and acetabular angle (Sharp angle) can not be detected. Therefore, X-ray examination is not recommended for children within 3 months, which is more reliable in children of 4-6 months when the ossification center of femoral head appears. With the application of digital radiography (DR) technology, digital X-ray imaging is fast, with high definition, low radiation, and strong post-processing function. Therefore, X-ray can become the preferred auxiliary method for the diagnosis and observation of DDH children of 4-6 months. Finally, compared with ultrasound and X-ray examination, the advantage of MRI is mainly reflected in the higher resolution in displaying the soft tissues, which is difficult to achieve by other examination methods. MRI can observe the pathological changes of soft tissue structure in and around the hip joints and then determine the progression and treatment of children diagnosed by ultrasound and X-ray examination in the early stage and constantly update data for subsequent treatment. In summary, MRI is mainly used to display the relationship between the femoral head and the acetabulum after closed reduction or open reduction and can display both cartilage and joint labrum. At the same time, sedation is required for children in this examination. Therefore, MRI is not suitable for children with low months of age.

The ROC curves showed that the diagnostic efficacy from high to low was the MRI + high-frequency ultrasound $+\mathrm{X}$-ray, high-frequency ultrasound $+\mathrm{X}$-ray, MRI, 
TABLE 1: Comparison of general data $(n=30)$.

\begin{tabular}{|c|c|c|c|c|}
\hline Observation indexes & Group X & Group Y & Group Z & $P$ \\
\hline $\begin{array}{l}\text { Children condition } \\
\text { Gender } \\
\text { Male } \\
\text { Female }\end{array}$ & $\begin{array}{l}14(46.67 \%) \\
16(53.33 \%)\end{array}$ & $\begin{array}{l}15(50 \%) \\
15(50 \%)\end{array}$ & $\begin{array}{l}13(43.33 \%) \\
17(56.67 \%)\end{array}$ & $<0.05$ \\
\hline $\begin{array}{l}\text { Injured sides } \\
\text { Left side } \\
\text { Right side } \\
\text { Bilateral sides } \\
\text { Birth weight }(\mathrm{kg}) \\
\end{array}$ & $\begin{array}{c}18(60 \%) \\
9(30 \%) \\
3(10 \%) \\
3.23 \pm 0.36 \\
\end{array}$ & $\begin{array}{c}17(56.67 \%) \\
8(26.67 \%) \\
5(16.67 \%) \\
3.25 \pm 0.37 \\
\end{array}$ & $\begin{array}{c}17(56.67 \%) \\
9(30 \%) \\
4(13.33 \%) \\
3.24 \pm 0.36 \\
\end{array}$ & $\begin{array}{l}<0.05 \\
<0.05 \\
<0.05 \\
<0.05\end{array}$ \\
\hline $\begin{array}{l}\text { Maternal risk factors } \\
\text { Breech presentation } \\
\text { Multiple pregnancy } \\
\text { Oligohydramnios }\end{array}$ & $\begin{array}{c}14(46.67 \%) \\
8(26.67 \%) \\
16(53.33 \%) \\
\end{array}$ & $\begin{array}{c}15(50 \%) \\
8(26.67 \%) \\
18(60 \%) \\
\end{array}$ & $\begin{array}{c}13(43.33 \%) \\
9(30 \%) \\
17(56.67 \%) \\
\end{array}$ & $\begin{array}{l}<0.05 \\
<0.05 \\
<0.05\end{array}$ \\
\hline
\end{tabular}

TABLE 2: Clinical diagnostic results in three groups $(n(\%))$.

\begin{tabular}{lccc}
\hline Observation indexes & Group X & Group Y & Group Z \\
\hline Acetabular dysplasia & $8(26.67)$ & $8(26.67)$ & $7(23.33)$ \\
Subluxation of the femoral head & $9(30)$ & $10(33.33)$ & $9(30)$ \\
Complete dislocation of the femoral head & $10(33.33)$ & $9(30)$ & $12(40)$ \\
\hline
\end{tabular}

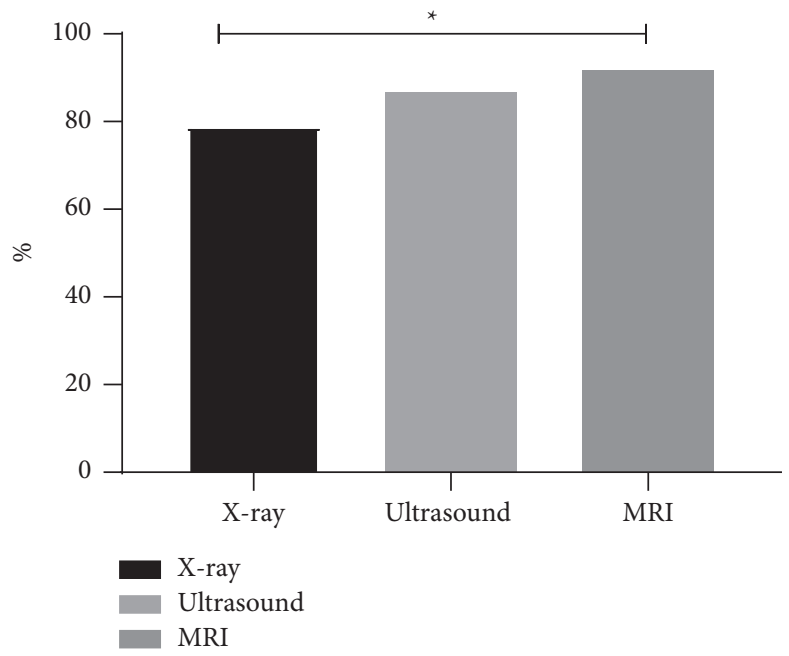

FIGURE 1: Diagnostic accuracy (\%). Note: the abscissa represented the imaging methods, and the ordinate represented the percentage (\%). $\mathrm{X}$-ray examination confirmed 71 cases $(78.89 \%)$ of DDH, ultrasound confirmed 78 cases (86.67\%), and MRI confirmed 55 cases (91.67\%). *indicated a notable difference in the diagnostic accuracy between X-ray and MRI $\left(X^{2}=4.640, P=0.031\right)$.

and X-ray. Therefore, this study believed that most of the hip joints of children at low months are cartilage components, and high-frequency ultrasound can better present the unossified hip joint structure. Early screening of $\mathrm{DDH}$ is achieved by observing the main structures of the femoral neck epiphyseal plate, lower edge of the iliac bone, synovial reflex, femoral head, cartilage top, turning point, joint capsule, labrum, and bone top in the ultrasound images, which provides a reliable basis for clinical diagnosis. X-ray examination can be used as a routine auxiliary examination for children over three months, which is conducive to further improving the clinical diagnosis rate of children at low age. MRI is of irreplaceable importance in older DDH children, which is more accurate in judging and evaluating the growth of the acetabulum, the pathological changes of the soft tissue structure in and around the hip joints, and the evolution process. It provides very important reference data for clinical diagnosis and treatment. MRI combined with high-frequency ultrasound and X-ray examination can provide more complete and reliable information for the diagnosis and treatment, thus better guiding clinical treatment, prognosis, and dynamic follow-up. However, this retrospective study lacked the follow-up information of some children. Therefore, the application value of $\mathrm{X}$-ray, high-frequency ultrasound, and MRI in the dynamic follow-up of children with DDH should be noted in the subsequent studies. 


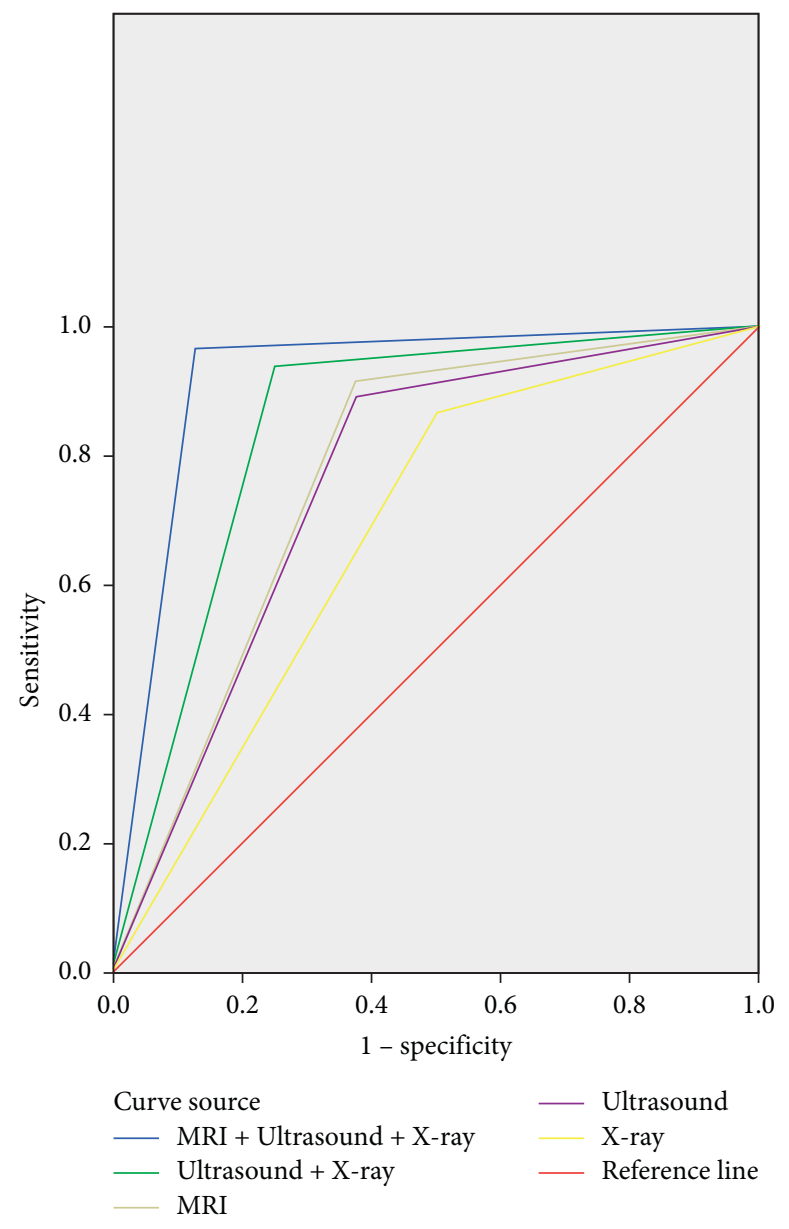

Figure 2: ROC curves.

TABle 3: Area under the curves.

\begin{tabular}{lcccc}
\hline Test result variables & Area & Standard error $^{\mathrm{a}}$ & Progressive Sig $^{\mathrm{b}}$ & Progressive 95\% confidence interval $^{\text {(n) }}$ \\
\hline MRI + ultrasound + X-ray & 0.919 & 0.069 & 0.000 & $0.000-1.000$ \\
Ultrasound + X-ray & 0.845 & 0.095 & 0.001 & $0.599-1.000$ \\
MRI & 0.770 & 0.106 & 0.012 & $0.563-0.977$ \\
Ultrasound & 0.758 & 0.105 & 0.017 & $0.551-0.964$ \\
X-ray & 0.659 & 0.111 & 0.140 & $0.441-0.876$ \\
\hline
\end{tabular}

a, under nonparametric hypothesis; $b$, null hypothesis, actual area $=0.5$.

In conclusion, ultrasound combined with X-ray has obvious advantages in the diagnosis of children at low months of age, while MRI has outstanding advantages in the diagnosis of children at high months of age. MRI combined with ultrasound and X-ray can significantly improve the diagnostic accuracy of DDH and provide objective data support for the clinical treatment of children.

\section{Data Availability}

Data to support the findings of this study are available upon reasonable request from the corresponding author.

\section{Conflicts of Interest}

The authors have no conflicts of interest to declare.

\section{References}

[1] S. W. Verbruggen, B. Kainz, S. C. Shelmerdine et al., "Altered biomechanical stimulation of the developing hip joint in presence of hip dysplasia risk factors," Journal of Biomechanics, vol. 78, pp. 1-9, 2018.

[2] M. Willey, H. Tai, H. Thomas-Aitken, and J. E. Goetz, "Diagnosis and management of borderline hip dysplasia and acetabular retroversion," The Journal of Hip Surgery, vol. 2, no. 4, pp. 156-166, 2018.

[3] M. Chen and X.-F. Shang, "Surgical treatment for young adult hip dysplasia: joint-preserving options," International Orthopaedics, vol. 40, no. 5, pp. 891-900, 2016.

[4] C. A. Barrera, S. A. Cohen, W. N. Sankar, V. M. Ho-Fung, R. W. Sze, and J. C. Nguyen, "Imaging of developmental dysplasia of the hip: ultrasound, radiography and magnetic resonance imaging," Pediatric Radiology, vol. 49, no. 12, pp. 1652-1668, 2019. 
[5] M. R. Schmitz, A. S. Murtha, and J. C. Clohisy, "Developmental dysplasia of the hip in adolescents and young adults," Journal of the American Academy of Orthopaedic Surgeons, vol. 28, no. 3, pp. 91-101, 2020.

[6] R. W. Paton and Q. Choudry, "Developmental dysplasia of the hip (DDH): diagnosis and treatment," Orthopaedics and trauma, vol. 30, no. 6, pp. 453-460, 2016.

[7] Y. Ishidou, K. Matsuyama, D. Sakuma et al., "Osteoarthritis of the hip joint in elderly patients is most commonly atrophic, with low parameters of acetabular dysplasia and possible involvement of osteoporosis," Archives of Osteoporosis, vol. 12, no. 1, p. 30, 2017.

[8] C. Tréguier, M. Chapuis, B. Branger et al., "Pubo-femoral distance: an easy sonographic screening test to avoid late diagnosis of developmental dysplasia of the hip," European Radiology, vol. 23, no. 3, pp. 836-844, 2013.

[9] C. Kerim Kursat, K. Hasan Erdinc, and S. Andac, "Segmentation of the ilium and femur regions from ultrasound images for diagnosis of developmental dysplasia of the hip," Journal of Medical Imaging and Health Informatics, vol. 6, no. 2, pp. 449-457, 2016.

[10] K. J. Donnelly, K. W. Chan, and A. P. Cosgrove, "Delayed diagnosis of developmental dysplasia of the hip in Northern Ireland," The Bone \& Joint Journal, vol. 97-B, no. 11, pp. 1572-1576, 2015.

[11] M. de Hundt, F. Vlemmix, B. W. J. Mol, and M. Kok, "Comment on: a meta-analysis of common risk factors associated with the diagnosis of developmental dysplasia of the hip in newborns," European Journal of Radiology, vol. 82, no. 1, p. 199, 2013.

[12] J. Mace and R. W. Paton, "Neonatal clinical screening of the hip in the diagnosis of developmental dysplasia of the hip," The Bone \& Joint Journal, vol. 97-B, no. 2, pp. 265-269, 2015.

[13] I. Swarup, C. L. Penny, and E. R. Dodwell, "Developmental dysplasia of the hip," Current Opinion in Pediatrics, vol. 30, no. 1, pp. 84-92, 2018.

[14] M. J. Anderton, G. R. Hastie, and R. W. Paton, “The positive predictive value of asymmetrical skin creases in the diagnosis of pathological developmental dysplasia of the hip," The Bone \& Joint Journal, vol. 100-B, no. 5, pp. 675-679, 2018.

[15] Q. A. Choudry and R. W. Paton, "Neonatal screening and selective sonographic imaging in the diagnosis of developmental dysplasia of the hip," The Bone \& Joint Journal, vol. 100-B, no. 6, pp. 806-810, 2018.

[16] G. Fatma Dilek, S. Aydin, E. Fatihoglu, E. Elif, and K. Pinar Nercis, "Optimizing the time for developmental dysplasia of the hip screening earlier or later?" Ultrasound Quarterly, vol. 35, no. 2, pp. 130-135, 2019.

[17] R. Agostiniani, G. Atti, S. Bonforte et al., "Recommendations for early diagnosis of Developmental Dysplasia of the Hip (DDH): working group intersociety consensus document," Italian Journal of Pediatrics, vol. 46, no. 1, p. 150, 2020.

[18] P. Touzopoulos and N. G. Markeas, "Asymmetrical thigh creases or isolated thigh crease may be a false positive sign with low predictive value in the diagnosis of developmental dysplasia of the hip in infants: a prospective cohort study of 117 patients," European Journal of Orthopaedic Surgery and Traumatology, vol. 30, no. 1, pp. 133-138, 2019.

[19] N. Padilla-Raygoza, G. Olvera-Villanueva, D. C. DelgadoSandoval Silvia, T. Cordova-Fraga, M. A. Sosa-Aquino, and V. Beltran-Campos, "Validity and reliability of electroacoustic probe for diagnosis of developmental dysplasia of the hip," BMC Pediatrics, vol. 17, no. 1, 2017.
[20] B. M. Barkatali, H. Imalingat, J. Childs, A. Baumann, and R. Paton, "MRI versus computed tomography as an imaging modality for postreduction assessment of irreducible hips in developmental dysplasia of the hip: an interobserver and intraobserver reliability study," Journal of Pediatric Orthopaedics B, vol. 25, no. 6, pp. 489-492, 2016.

[21] B. A. Shaw and L. S. Segal, "Evaluation and referral for developmental dysplasia of the hip in infants," Pediatrics, vol. 138, no. 6, Article ID e20163107, 2016.

[22] N. Quader, E. K. Schaeffer, A. J. Hodgson, R Abugharbieh, and K Mulpuri, "A systematic review and meta-analysis on the reproducibility of ultrasound-based metrics for assessing developmental dysplasia of the hip," Journal of pediatric orthopedics, vol. 38, no. 6, pp. E305-E311, 2018.

[23] P. Walbron, F. Müller, L. Mainard-Simard, A. Luc, and P. Journeau, "Bone maturation of MRI residual developmental dysplasia of the hip with discrepancy between osseous and cartilaginous acetabular index," Journal of Pediatric Orthopaedics B, vol. 28, no. 5, pp. 419-423, 2019.

[24] N. Alassaf, "Single-leg spica provides adequate stability after open reduction in developmental dysplasia of the hip," Archives of Orthopaedic and Trauma Surgery, vol. 138, no. 2, pp. 173-178, 2018. 